\title{
Rappels technologiques, biais méthodologiques possibles et conditions de mesure
}

\author{
Jacques Auger
}

CECOS PARIS-BICETRE, Centre Hospitalier de Bicêtre, 94275 Le Kremlin-Bicêtre cedex. ; Nouvelle adresse : CECOS PARIS-COCHIN, Laboratoire d'Histologie Embryologie orienté en Biologie de la Reproduction, Groupe Hospitalier Cochin Port-Royal, 123, Boulevard de Port-Royal, 75014, PARIS

\section{RESUME}

De nombreux facteurs peuvent invalider les résultats d'une analyse du mouvement des spermatozoïdes par les systèmes de microvidéographie assistée par ordinateur : une standardisation de l'analyse est donc indispensable. Des notions de base de la technologie de ces systèmes, une bonne connaissance de la physiologie spermatique et une rigueur dans toutes les étapes de l'analyse sont des pré-requis essentiels pour garantir une analyse fiable car ces systèmes ne sont pas des automates "prêt à l'emploi".

Mots-clés : spermatozoïdes humains ; microvidéographie assistée par ordinateur ; mouvement ; mobilité ; analyse standardisée.

\section{INTRODUCTION}

Les systèmes d'analyse des spermatozoïdes par microvidéographie assistée par ordinateur ont été développés pour tenter de pallier le caractère imprécis et/ou subjectif de l'analyse manuelle du sperme $[4,21]$. Ils permettent la mesure de paramètres du mouvement fondés sur la cinématique des têtes des spermatozoïdes et non des flagelles. Certains de ces paramètres présentent une valeur diagnostique et pronostique dans les situations d'infertilité masculine lors d'évaluations in vivo et in vitro $[7,10$,
$11,22$; revue dans 1$]$. Ces systèmes se sont imposés par rapport à des techniques plus anciennes et plus lourdes telles la microphotographie $[13,18]$ ou la microcinématographie $[5,20]$ grâce à leurs possibilités de mesures en temps réel et leur relative simplicité d'emploi.

Un système d'analyse des spermatozoïdes par ordinateur comprend un microscope équipé d'optiques de contraste de phase permettant le meilleur contraste des têtes des spermatozoïdes présents dans le champ microscopique, l'analyse ultérieure dépendant de leur détection pendant une durée d'acquisition donnée. Le microscope est équipé d'une platine thermostatée. Les images à analyser sont soit enregistrées sur bande vidéo pour une analyse différée soit directement traitées par le système composé d'un microordinateur de forte capacité doté d'un disque dur et de logiciels et matériels dédiés à l'analyse d'images séquentielles.

\section{FORMATION, RESTITUTION DE L'IMAGE ET PRECALIBRATION DU SYSTEME AVANT TOUTE ANALYSE}

L'image du champ microscopique de la préparation posée sous l'objectif est formée sur la surface sensible de la caméra vidéo. Avec les caméras CCD, les plus utilisées actuellement, l'image du champ microscopique est discrétisée en un ensemble de points élé- 
mentaires ou pixels, de façon analogique aux grains d'argent composant une image photographique ; à chaque point correspond un voltage donné proportionnel à l'intensité lumineuse reçue et l'ensemble des signaux correspondant à tous les pixels est codé sous la forme d'un signal analogique complexe pour stockage, transmission et visualisation. Les pixels composant l'image sont arrangés sous la forme d'une surface de 512 x 512 pixels. Cette technique ne permet pas une visualisation directe sur l'écran du moniteur.

Selon la technologie vidéo standard, l'image est restituée sur l'écran du moniteur en 1/30ème de seconde (normes américaines) : le premier champ une fois affiché reste illuminé tandis que le second s'affiche et permet la restitution de l'image totale. Cette technologie est conforme à la vision humaine et peu coûteuse. La fréquence d'acquisition des positions successives des têtes est donc $30 \mathrm{~Hz}$ en pleine résolution ou $60 \mathrm{~Hz}$ en utilisant un champ vidéo sur 2 avec un décalage d'une ligne. Certaines caméras couplées à des dispositifs de numérisation et de restitution des images permettent des acquisitions à des fréquences supérieures, $100,200 \mathrm{~Hz}$ et plus, offrant la possibilité d'une analyse fine des mouvements flagellaires pour des espèces et des situations expérimentales variées.

L'étape suivante avant toute analyse est la numérisation de l'image suivie du seuillage. La numérisation consiste à affecter un nombre entier à chaque point élémentaire de l'image selon une échelle de 256 niveaux de gris par exemple, du blanc (niveau 0), au noir (niveau 255). Le seuillage ou détection permet une partition de l'image ne retenant que les informations d'interêt, c'est à dire les coordonnées des points-image définissant les têtes des spermatozoïdes ou leurs contours. En contraste de phase, ces derniers correspondent aux zones de plus forte transition de niveaux de gris.

Une précalibration du système définissant les tailles minimum des têtes des spermato- zoïdes est essentielle car des débris (taille inférieure) ou d'autres cellules (taille supérieure) présents dans le champ microscopique peuvent présenter des niveaux de gris similaires ou les mêmes transitions de contraste par rapport au fond de la préparation.

\section{ACQUISITION DES POSITIONS SUCCESSIVES DES TETES, RECONSTRUCTION DES TRAJECTOIRES ET CALCUL DES PARAMETRES DU MOUVEMENT}

A partir des coordonnées des bords ou de l'intensité de l'ensemble des pixels composant les têtes des spermatozoïdes présents dans le champ, le logiciel calcule la position du centre de gravité (centroïde) de chaque tête. Ces calculs sont effectués en temps réel et les coordonnées séquentielles de la position des centroïdes, champ après champ, selon la fréquence d'acquisition choisie sont envoyées sur le disque dur sous la forme d'une chaîne de coordonnées chronologiques.

D'un champ vidéo au suivant, le logiciel effectue la reconstruction des trajectoires des spermatozoildes pendant une période d'acquisition prédéfinie en joignant les positions des centroïdes des têtes des spermatozoïdes présentes dans les champs vidéo successifs. Cette étape est réalisée en spécifiant au système les déplacements dans l'espace minimum et maximum qu'un spermatozoïde peut effectuer d'un champ vidéo au suivant. Compte-tenu de son principe, cet algorithme ne réussit pas toujours à bien reconstruire des trajectoires qui se coupent. En effet, la fréquence de croisement est proportionnelle au produit de la vitesse moyenne des spermatozoïdes par la concentration, la longueur moyenne des têtes et la durée de l'acquistion [4]. Une dilution des spermes présentant une concentration supérieure à 40 millions par $\mathrm{ml}$ permet de minimiser ce problème [4]. A partir des données enregistrées pour chaque cellule, le logiciel calcule ensuite des paramètres clas- 
siques tels que la concentration spermatique ou le pourcentage de spermatozoïdes mobiles et la valeur moyenne de paramètres standardisés relatifs à la vigueur de déplacement des cellules et à la forme de leur trajectoire [17].

\section{FACTEURS MODULANT LES RESULTATS DE L'ANALYSE}

De nombreux facteurs peuvent invalider les résultats de l'analyse. Ils peuvent être liés à l'état physiologique des cellules, à l'interaction entre l'observateur et le système ou encore à des contraintes technologiques inhérentes au système.

La température $[2,19]$ ou le milieu environnant $[8,12,14,15]$ ont un effet bien connu sur le mouvement des spermatozoïdes. Nous avons précédemment montré que l'augmentation de la vitesse de progression linéaire des spermatozoides humains avec la température est de l'ordre d'un $\mu \mathrm{m} / \mathrm{s} /{ }^{\circ} \mathrm{C}$ [2]. Dans le plasma séminal la valeur moyenne de l'ensemble des paramètres mesurés augmente significativement en fonction de la température ; un phénomène similaire est observé après centrifugation sur gradient de percoll et incubation d'au moins une demi-heure dans un milieu capacitant et les différences entre les deux types d'environnement sont également impor- tantes (Tableau 1) : les paramètres du mouvement, à l'exception de la linéarité qui est plus basse, ont des valeurs moyennes très supérieures après élimination du plasma séminal et resuspension dans un milieu capacitant. Ces résultats indiquent la nécessité d'effectuer les analyses à des températures fixes et d'adapter la précalibration des systèmes aux conditions environnementales des spermatozoïdes.

La fréquence d'acquisition joue un rôle fondamental [16]. La durée d'acquisition joue également un rôle important : à $60 \mathrm{~Hz}$ une acquisition pendant 1/6éme de seconde ne permet pas d'obtenir des résultats valides notamment de ALH et VSL (définitions des paramètres données dans le tableau 1); au contraire, l'acquisition sur des périodes de $1 / 2$ s à $3 / 4$ de seconde permet d'obtenir une évaluation correcte du comportement cinétique des spermatozoïdes.

La méthode de calcul de la vitesse moyenne et de l'amplitude qui dépend des systèmes et n' est pas contrôlable par l'opérateur modifie sensiblement les valeurs calculées de ces paramètres. Dans la méthode "de lissage fixe" la valeur de ALH est calculée à partir des valeurs moyennes des distances orthogonales de la trajectoire à la trajectoire moyenne. Dans la méthode de "lissage adaptatif", le tracé de la trajectoire moyen-

Tableau 1 : Effets de la température et du milieu de suspension (sperme frais ou resuspendu dans un milieu capacitant, après centrifugation sur gradient de percoll) des spermatozoüdes sur la valeur moyenne des paramètres du mouvement (mesures à partir d' une bande vidéo, au moins 200 cellules analysées à $60 \mathrm{~Hz}$ pendant $0,5 s$ dans une microcellule de $20 \mathrm{\mu m}$ de profondeur; Système CTS, Motion Analysis Co).(Données personnelles non publiées).

\begin{tabular}{lcccc}
\hline & \multicolumn{2}{c}{ Sperme frais } & \multicolumn{2}{c}{ milieu B2 } \\
\cline { 2 - 3 } $\begin{array}{l}n n n y y \\
\text { Paramètres }\end{array}$ & $\mathbf{2 5}^{\circ} \mathbf{C}$ & $\mathbf{3 7}^{\circ} \mathbf{C}$ & $\mathbf{2 5}^{\circ} \mathbf{C}$ & $\mathbf{3 7}^{\circ} \mathbf{C}$ \\
\hline VSL & 24 & 38 & 32 & 50 \\
VCL & 61 & 85 & 96 & 119 \\
LIN & 40 & 46 & 34 & 41 \\
ALH & 3,1 & 4,1 & 5,4 & 6,2 \\
\hline
\end{tabular}

* selon les normes de l' OMS (17), VSL : vitesse de progression linéaire, $\mu \mathrm{m} / \mathrm{s}$; VCL : vitesse curvilinéaire, $\mu \mathrm{m} / \mathrm{s}$; LIN : linéarité de trajectoire, \%; ALH : amplitude de débattement latéral de la tête, $\mu m$. 
ne et le calcul de ALH sont beaucoup plus dépendants des variations instantanées de forme de la trajectoire : il existe ainsi une correspondance parfaite entre les points de la trajectoire réelle et les points de la trajectoire moyenne et le calcul de ALH ne repose plus sur des mesures de distances orthogonales mais sur des mesures de vecteurs de tailles variées : cette dernière méthode rend mieux compte de l'hétérogénéité cinétique des populations spermatiques analysées.

C'est l'observateur qui définit le seuil de détection des spermatozoïdes quelle que soit la méthode algorithmique employée. Le seuil fait varier de façon importante les valeurs des paramètres mesurés comme l'indique le tableau 2. Par exemple, la concentration est sous estimée avec un seuil trop élevé (sous détection), des spermatozoïdes ne sont pas comptés ; inversement, un seuil trop bas (surdétection) induit des artefacts électroniques qui surestiment le nombre de particules présentes dans le champ et donc la concentration. Ces artefacts sont temporellement instables et peuvent être pris pour des spermatozoïdes mobiles sur place ce qui à pour conséquence de surestimer le pourcentage de spermatozoïdes mobiles comme l'indique le tableau 2 ; les paramètres du mouvement sont égale- ment affectés par une détection incorrecte.

Le choix de la chambre d'analyse utilisée revêt également une grande importance. Il a été montré que la chambre de Makler fausse les mesures de concentration et contraint le mouvement des spermatozoïdes [9]. Dans notre expérience, avec des microcellules ( $\mu$ cell; Fertility Technology) de 12 microns d'épaisseur, la vitesse curvilinéaire est surestimée, probablement à cause de la contrainte du débattement flagellaire. Par ailleurs, l'utilisation de chambres trop profondes $(50 \mu \mathrm{m})$ résulte en une hétérogénéité de distribution des spermatozoïdes dans la profondeur : il est possible que dans de telles chambres les calculs soient biaisés, les valeurs des paramètres mesurés pouvant ne caractériser qu'une sous population de spermatozoïdes différente du reste de la population ne se trouvant pas dans le plan focal. De plus le risque d'exclure des trajectoires du fait de la possibilité d'un mouvement tridimensionnel plus marqué (en particulier lors de la capacitation) est certainement plus grande. Selon un expertise de l'OMS, des chambres d'analyse d'une profondeur de 20 microns semblent adéquates pour éviter ces problèmes pour les spermes frais et préparés [17].

Tableau 2 : Effets du choix du seuil de détection des têtes des spermatozoüdes, S, (2000 niveaux de gris, $0=$ blanc, 1999 = noir). (sperme frais, mesures à partir d'une bande vidéo, au moins 200 cellules analysées à $60 \mathrm{~Hz}$ pendant $0,5 \mathrm{~s}$ dans une microcellule de 20 $7 \mathrm{~m}$ de profondeur; Système CTS, Motion Analysis Co).(Données personnelles non publiées).

\begin{tabular}{|c|c|c|c|}
\hline Paramètres mesurés* & $\begin{array}{c}\mathrm{S}=1210 \\
\text { sous-détection }\end{array}$ & $\begin{array}{l}\mathrm{S}=1066 \\
\text { détection correcte }\end{array}$ & $\begin{array}{c}\mathrm{S}=992 \\
\text { surdétection }\end{array}$ \\
\hline Nombre d'éléments compté & 137 & 203 & 352 \\
\hline $\mathrm{CON}$ & 11 & 17 & 32 \\
\hline MOT & 47 & 52 & 68 \\
\hline $\mathrm{VSL}^{* *}$ & 37 & 33 & 28 \\
\hline $\mathrm{VCL}^{* *}$ & 81 & 77 & 78 \\
\hline $\mathrm{LIN}^{* *}$ & 49 & 46 & 35 \\
\hline $\mathrm{ALH}^{* *}$ & 4,0 & 3,7 & 5,1 \\
\hline
\end{tabular}

* selon les normes de l' OMS (17), CON : concentration, $x 106 / \mathrm{ml} ;$ MOT : pourcentage de spermatozö̈des mobiles ; VSL : vitesse de progression linéaire, $\mu \mathrm{m} / \mathrm{s} ; \mathrm{VCL}$ : vitesse curvilinéaire, $\mu \mathrm{m} / \mathrm{s} ; \mathrm{LIN}$ : linéarité de trajectoire, \% ALH : amplitude de débattement latéral de la tête, $\mu m .{ }^{* *}$ valeur moyenne. 
COMPARAISONS DES RESULTATS OBSERVATEUR/SYSTEME ET ENTRE SYSTEMES

De tels systèmes sont réputés ne pas fournir des mesures correctes de la concentration spermatique. Nous avons étudié le degré de concordance entre mesure manuelle et mesure automatisée (Figure 1A) : sur une large gamme de concentrations, la corrélation est assez satisfaisante et il n'existe pas de différence significative entre les valeurs moyennes fournies par les deux méthodes. Il est intéressant de noter qu'avec le système employé (CTS, Motion Analysis Co), un contrôle de la taille du champ analysé aboutissant à une "dilution électronique" par une réduction ajustable de la taille du champ autorise des mesures sans dilution physique pour des spermes très concentrés (Figure 1A); cependant, cette méthode ne résoud pas pour autant le A

MANUEL $96,1(25,9)$

CTS $100,6(22,6)$

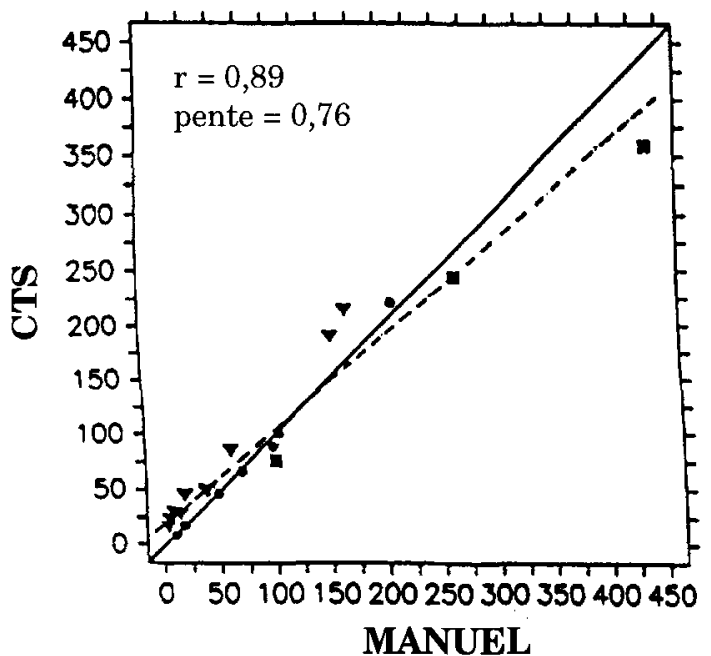

problème des croisements de trajectoires. La corrélation entre résultats fournis par 2 systèmes, HTM (Hamilton-Thorn) et CTS, pour la concentration - après dilution si la concentration excède 40 millions par $\mathrm{ml}$ et moyennant une standardisation très rigoureuse de l'analyse - est très bonne et les valeurs moyennes ne differrent pas significativement des valeurs trouvées par l'analyse manuelle [6] (Figure 1B). Entre machines, il existe une différence significative des valeurs moyennes de concentration ; cette différence est peu importante et selon les auteurs de cette étude, elle n'a probablement pas de signification biologique [6]. La corrélation entre résultats de l'analyse de la mobilité, manuelle et par le CTS (Figure $2 \mathrm{~A}$ ), est moins bonne que pour la concentration. Davis et KaTZ ont obtenu des résultats similaires [6] (Figure 2B). Par contre la concordance des résultats obtenus par les machines HTM et CTS est remarquable. B

$\begin{array}{lll}\text { MANUEL } & \text { CTS } & \text { HTM } \\ 58,1(7,2) & 61,2(7,0)^{*} & \mathbf{5 7 , 8}(7,0)^{* *} \mathbf{P}<\mathbf{0 , 0 5}\end{array}$

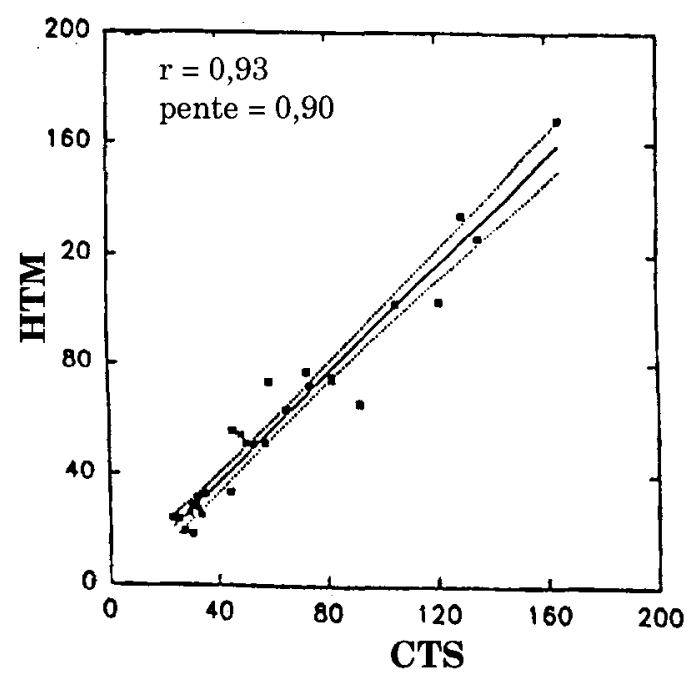

Figure $1:$ A. Comparaison des valeurs moyennes (ESM) de la concentration en spermatozoïdes $\left(x 10^{6 / m l}\right)$ par mesure manuelle et automatisée (CTS, Motion Analysis Co, $30 \mathrm{~Hz}$, analyse directe sans dilution) et corrélation entre méthodes ( $n=19$; données personnelles non publiées). B. Comparaison des valeurs moyennes (ESM) de la concentration en spermatozoïdes $\left(x 10^{6} / \mathrm{ml}\right)$ par mesure manuelle et automatisée (CTS, Motion Analysis Co ; HTM, Hamilton Thorn) et corrélation entre machines, d'après Davis et Katz $\left(6 ; n=30,30 \mathrm{~Hz}\right.$, bandes vidéo, dilution si concentration $\left.>40 \times 10^{6} / \mathrm{ml}\right)$. 
A

MANUEL

$29,2(3,4)$

CTS

$24,1(3,2)$

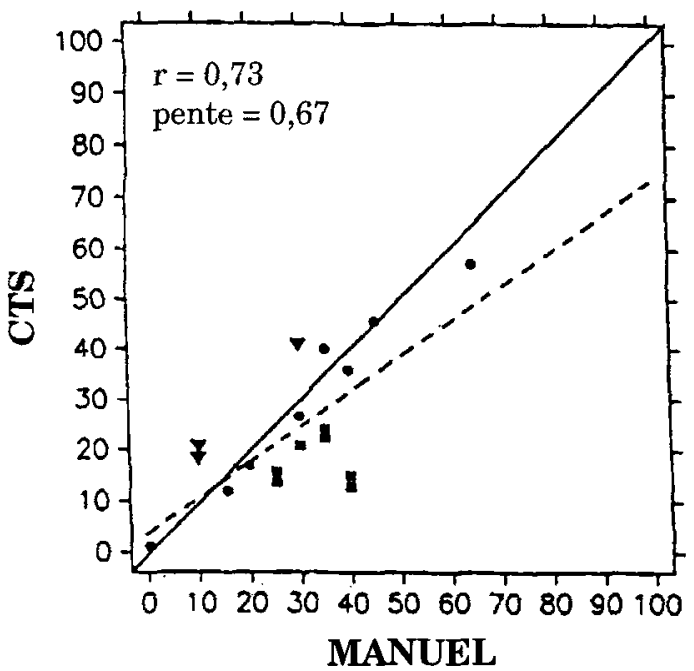

B

MANUEL CTS HTM

NS $\quad 51,1(2,5), \dagger 38,7(2,5)^{*} 41,8(2,7) \dagger^{*}, \dagger \mathbf{P}<\mathbf{0 , 0 5}$

Figure 2 : A. Comparaison des valeurs moyennes (ESM) du pourcentage de spermatozoïdes mobiles par mesure manuelle et automatisée (CTS, Motion Analysis Co, $30 \mathrm{~Hz}$, analyse directe sans dilution) et corrélation entre méthodes ( $n=19$; données personnelles non publiées). B. Comparaison des valeurs moyennes (ESM) du pourcentage de spermatozoïdes mobiles par mesure manuelle et automatisée (CTS, Motion Analysis Co ; HTM, Hamilton Thorn) et corrélation entre machines, d'après Davis et Katz $(6 ; n=30,30 \mathrm{~Hz}$, bandes vidéo, dilution si concentration $>40 \times 10^{6} / \mathrm{ml}$ ).

Concernant les paramètres du mouvement, sous réserve d'une standardisation très rigoureuse de la calibration de chaque machine, KATZ et Davis ont montré une bonne concordance des résultats entre les 2 machines [6] (Figure 3 A-B-C) avec toutefois une valeur de ALH systématiquement plus élevée avec le système HTM qu'avec le système CTS, cet écart étant probablement dû à la différence des algorithmes pour leur calcul dans les versions des 2 machines utilisées [6]. Nous avons trouvé des résultats divergents pour certains des paramètres mesurés comparant 3 systèmes (HTM, CTS et le système français Speed Sperm, JCD International) dont les versions présentaient des différences matérielles et logicielles importantes [3].

\section{RECOMMANDATIONS POUR UNE ANALYSE STANDARDISEE}

Toutes ces données indiquent qu'une standardisation rigoureuse de l'analyse est un pré-requis indispensable pour obtenir des résultats fiables. Des recommandations dans le but d'analyse standardisée sont proposées (Tableau 3).

\section{CONCLUSION}

Une standardisation de l'analyse du sperme par les systèmes actuels de microvidéographie assistée par ordinateur est indispensable. Des notions de base de la technologie de ces systèmes, une bonne connaissance de la physiologie spermatique et une rigueur dans toutes les étapes de l'analyse sont des pré-requis essentiels pour garantir une ana- 


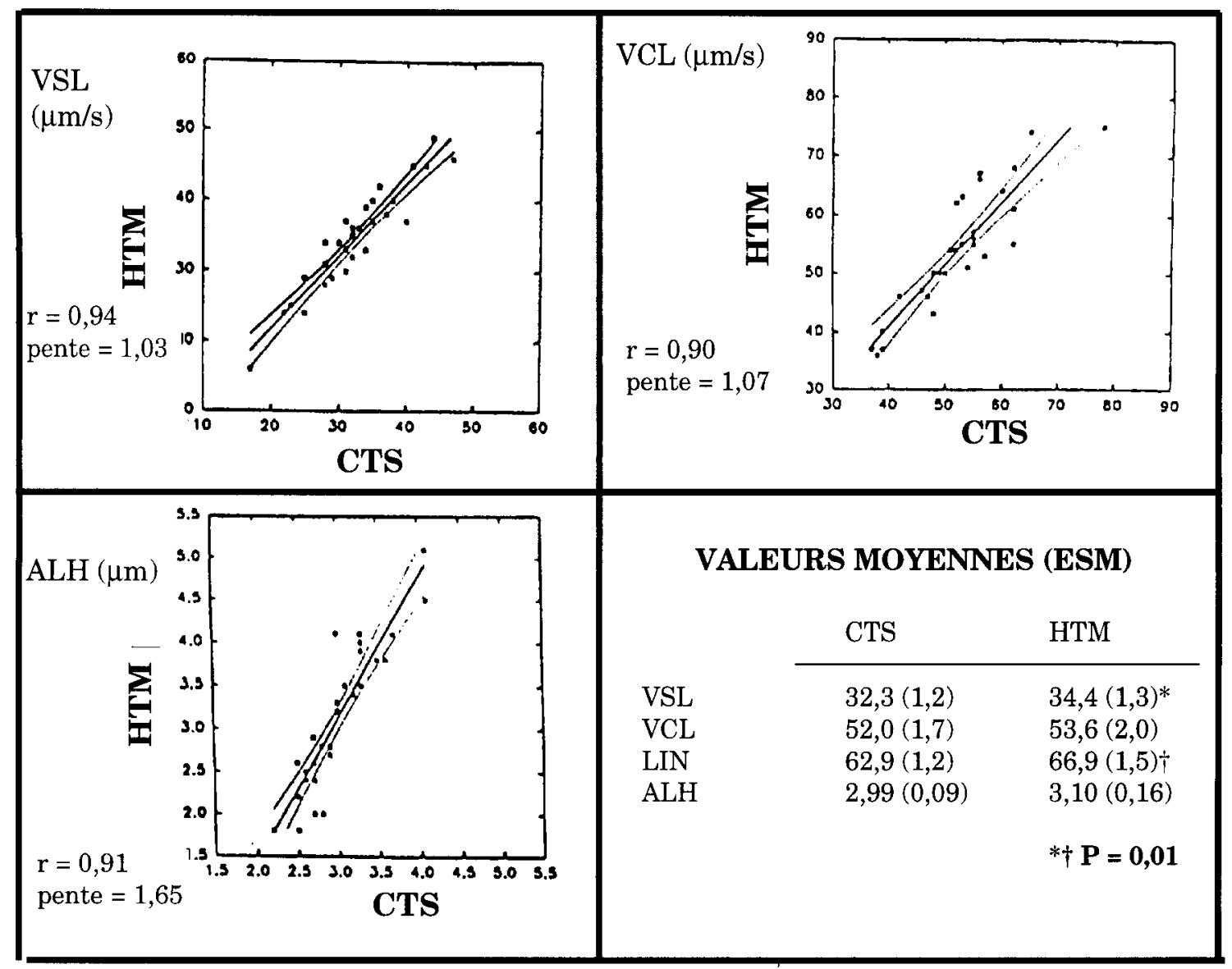

Figure 3 : Corrélation entre machines (HTM, Hamilton Thorn et CTS, Motion Analysis Co) des mesures de la vitesse de progression linéaire (VSL), de la vitesse curvilinéaire (VCL) et de l'amplitude de débattement de la tête (ALH) et comparaisons des valeurs moyennes (ESM) par les 2 machines, d'après Davis et Katz $(6 ; n=30,30 \mathrm{~Hz}$, bandes vidéo, dilution si concentration > $40 \times 10^{6} / \mathrm{ml}$ ).

lyse fiable car ces systèmes ne sont pas des automates "prêt à l'emploi". Dans leur état actuel, sous réserve d'une bonne pratique, ces systèmes sont utiles pour l'analyse de routine du sperme humain (concentration/ mobilité) et indispensables pour l'évaluation de la fonction cinétique en routine et en recherche. La technologie de ces systèmes reposant sur la vision par ordinateur évolue comme l'illustrent les développements actuels, matériel et logiciel. Elle offre un potentiel intéressant d'applications pour le laboratoire d'andrologie, tant pour l'analyse de routine (morphologie, analyse de spermes décongelés) que pour des analyses de la qualité fonctionnelle des spermato- zoïdes (taux de réaction acrosomique, quantification de marqueurs, ...).

\section{REFERENCES}

1. Auger J., Serres C., Wolf J.P., Jounannet P. : Mouvement des spermatozoïdes et fécondation. Contracept. Fertil. Sex., 1994, $22: 316-320$.

2. Auger J., Serres C., Feneux D. : Motion of individual spermatozoa, both normal and lacking the dynein arms, during a continuous temperature rise. Cell Motil. Cytoskeleton, 1990, 16 : 22-32.

3. Auger J., Sonnet M.L., Jounnnet P. : Automatic analysis of human sperm movement : comparative study of three CASA systems. Human Reprod., 1991, suppl, abstracts from the 7 th World Congress on IVF and assisted Procreation, p 338. 
1. prélèvement de sperme au laboratoire, maintien à température contrôlée $\left(37^{\circ} \mathrm{C}\right)$ jusqu' à l'analyse ( $\left.37^{\circ} \mathrm{C}\right)$,

2. analyse en contraste de phase dans l'heure qui suit le prélèvement (pour un sperme préparé sur gradient de percoll, analyse au minimum $1 / 2 \mathrm{~h}$ apres centrifugation) dans une chambre prévue à cet effet (épaisseur de la préparation $=20 \mu \mathrm{m}$ ),

3. si concentration $50 \times 10^{6} / \mathrm{ml}$, diluer dans du PBS, éventuellement dans du plasma séminal homologue,

4. enregistrer sur bande vidéo les spermes à analyser (contrôle de qualité de l'analyse),

5. ajuster les seuils de vitesse et les seuils de taille pour l'élimination des débris et autres cellules de façon optimale (intérêt des bandes vidéo et de la fonction "playback"),

6. analyse sur des périodes d'une demie seconde au moins, à $30 \mathrm{~Hz}$ pour le sperme frais, à $60 \mathrm{~Hz}$ pour des spermatozoïdes dans un milieu capacitant,

7. seuil de détection choisi de telle manière que mesures de concentration manuelle et par le système soient identiques (après préparation, une très légère surdétection peut être utile pour "pister" des spermatozoides présentant de faibles déviations hors du plan focal),

8. analyse de 100 cellules mobiles au moins par échantillon (200 si possible),

9. méthode standardisée de balayage de la préparation (en méandre, champs contigus, par exemple),

10. toujours imprimer, en plus des moyennes, les histogrammes des différents paramètres, leur distribution n'étant pas toujours normale.

4. Boyers S.P., Davis R.O., Katz D.F. : Automated semen analysis. Curr. Probl. Obstet. Gynecol. Fertil. $1989,12: 165-200$.

5. David G., Serres C., Jounnnet P. : Kinematics of human spermatozoa. Gamete Res., 1981, 4 : 83-95.

6. DAvis R.O., KATZ D.F. : Standardization and Comparability of CASA instruments. J. Androl., 1992, $13: 81-86$.

7. Feneux D., Serres C., Jouannet P. : Sliding spermatozoa : a dyskinesia responsible for human infertility ? Fertil. Steril., 1985, 44 : 508-514.

8. Ginsburg K.A., Sacco A.G., Moghissi K.S., SoroVETZ S. : Variations of movement characteristics with washing and capacitation of sperm. I. Univariate statistical analysis and detection of sperm hyperactivation. Fertil. Steril., 1989, $51:$ 869-873.

9. Ginsburg K.A., ARMant D.R. : The influence of chamber characteristics on the reliability of sperm concentration and movement measurements obtained by manual and videomicrographic analysis. Fertil. Steril., 1990, $53:$ 882-887.

10. Jeulin C., Feneux D., Jouannet P., Gulllet-Rosso F. Belaisch-Allart J., Frydman R., Testart J. : Sperm factors related to failure of human in vitro fertilization. J. Reprod. Fert., 1986, 76 : 735-742.
11. Jouannet P., Escalier D., Serres C., David G. : Motility of human spermatozoa without outer dynein arms. J. Submicrosc. Cytol., 1983, $15:$ 67-71.

12. MACK S.O., WolF D.P., TASH J.S. : Quantitation of specific parameters of motility in large numbers of human sperm by digital image processing. Biol. Reprod., 1988, 38 : 270-281.

13. MAKLER A. : Use of the elaborated multiple exposure photography (MEP) method in routine sperm motility analysis and for research purposes. Fertil. Steril., 1980, $33: 160-166$.

14. Morales P., Overstreet J.W., Katz D.F. : Changes in human sperm motion during capacitation in vitro. J. Reprod. Fert., 1988, 83 : 119-128.

15. Mortimer S.T., Mortimer D. : Kinematics of human spermatozoa incubated under capacitating conditions. J. Androl., 1989, $11:$ 195-203.

16. Mortimer D., Serres C., Mortimer S.T., Jounnnet P. : Influence of image sampling frequency on the perceived movement characteristics of progressively motile human spermatozoa. Gamete Res., 1988, 20 : 313-327.

17. Organisation Mondiale de la Santé : Manuel de laboratoire de l'OMS. Analyse du sperme humain et de l'interaction des spermatozoïdes avec le mucus cervical. Paris, Editions INSERM, 1993, 99-103. 
18. Overstreet J.W., Katz D.F., Hanson F.W., FonseCA J.R. : A simple inexpensive method for objective assessment of human sperm movement characteristics. Fertil. Steril., 1979, 31 : 162-172.

19. Rikmenspoel R. : Movements and active movements of bull sperm flagella as a function of temperature and viscosity. J. Exp. Biol., 1984, 108 : 205230 .

20. Schoevaert-Brossault D. : Automated analysis of human sperm motility. Comput. Biomed. Res., 1984, $17: 362-371$.

21. Walker J.S., Wineet H., Freund M. : A comparison of subjective and objective sperm motility evaluation. J. Androl., 1982, $3: 184-192$.

22. Wolf J.P., Feneux D., Escalier D., Rodrigues D., Frydman R., Jounnnet P. : Pregnancy after subzonal insemination with spermatozoa lacking outer dynein arms. J. Reprod Fert., 1993, $97: 487-492$.

ABSTRACT
Technology, methodological bias and
guidelines for standard procedures
Jacques AUGER
Computer-aided sperm analysis
(CASA) was developed in the last deca-
de to overcome the problems related to
the inherent subjectivity of manual
semen analysis. A typical CASA system
includes a video camera, a microscope
equiped with phase optics and a war-
ming stage, a microcomputer with
hardware and software dedicated to
motion analysis, a monitor and a prin-
ter. The image of the sperm cells under
the microscope is transformed into dis-
crete pixels producing a voltage pro-
portional to the intensity of the light
as light strikes the camera's CCD
array. In standard video technology,
the first field (set of rows; odd lines) is

scanned in one sixtieth of a second and remains illuminated, then the alternate set of rows (even lines) is scanned in the next one sixtieth of a second to display the complete video frame which requires one thirthieth of a second. The first step called digitization is the encoding of video pixels as numbers following a gray level scale between white and black. From this process the detection of the sperm heads in the field is performed and repeated on a field by field basis. The centroïd coordinates of the sperm heads in the field are calculated and sequentially transmitted to the hard disk. A path finder algorithm connects the centroids through time allowing the reconstruction of trajectories and the calculation of various parameters. Many factors modulating CASA measurements can invalidate the results of analysis. Consequently, a standardization of CASA is necessary. Various guidelines for accurate analysis are proposed.

In summary, basic knowledge on sperm physiology, CASA technology and rigorous standardized conditions of analysis are required for providing reliable and accurate results because CASA systems are not ready-to-use "robots". CASA is useful for routine semen analysis and essential for the measurement of sperm kinematics for clinical as well as for research pur. poses.

Key-words : computer aided sperm analysis ; microvideography; standard procedures ; human sperm 